\title{
Exact exponent in the remainder term of Gelfond's digit theorem in the binary case
}

by

\author{
Vladimir Shevelev (Beer-Sheva)
}

1. Introduction. For integers $m>1$ and $a \in[0, m-1]$, define

$$
T_{m, a}^{(j)}(x)=\sum_{0 \leq n<x, n \equiv a \bmod m, s(n) \equiv j \bmod 2} 1, \quad j=1,2,
$$

where $s(n)$ is the number of 1's in the binary expansion of $n$. Gelfond [7] proved that

$$
T_{m, a}^{(j)}(x)=\frac{x}{2 m}+O\left(x^{\lambda}\right), \quad j=0,1,
$$

where

$$
\lambda=\frac{\ln 3}{\ln 4}=0.79248125 \ldots
$$

This is the binary case of Gelfond's main digit theorem about the distribution of digit sums of arbitrary base in different residue classes. Gelfond's theorem initiated a whole line of research (see Notes on Chapter 3 in [1], as well as [10], [3], [9]). A related circle of works, dealing with the so-called Newman-like phenomena, started with the unexpected results of D. J. Newman [11] (see also [2], [5], [15]; again, an extensive bibliography may be found in [1]). In this paper, we shall be concerned only with the binary case of Gelfond's digit theorem. Recently, the author proved [13] that the exponent $\lambda$ in the remainder term in (2) is the best possible when $m$ is a multiple of 3 and is not the best possible otherwise. In this paper we give a simple formula for the exact exponent in the remainder term of (2) for an arbitrary $m$. Our method is based on constructing a recursion relation for the Newman-like sum corresponding to (1),

$$
S_{m, a}(x)=\sum_{0 \leq n<x, n \equiv a \bmod m}(-1)^{s(n)} .
$$

2000 Mathematics Subject Classification: Primary 11A63.

Key words and phrases: cyclotomic cosets of 2 modulo $m$, order of 2 modulo $m$, difference equation, trigonometric sums. 
It is sufficient for our purposes to deal with odd numbers $m$. Indeed, it is easy to see that, if $m$ is even, then

$$
S_{m, a}(2 x)=(-1)^{a} S_{m / 2,\lfloor a / 2\rfloor}(x) .
$$

For $m>1$ odd, consider the number $r=r(m)$ of distinct cyclotomic cosets of 2 modulo $m$ [8, pp. 104-105]. E.g., $r(15)=4$, since for $m=15$ we have the following four cyclotomic cosets of $2:\{1,2,4,8\},\{3,6,12,9\},\{5,10\}$, $\{7,14,13,11\}$.

Note that, if $C_{1}, \ldots, C_{r}$ are all different cyclotomic cosets of 2 modulo $m$, then

$$
\bigcup_{j=1}^{r} C_{j}=\{1, \ldots, m-1\}, \quad C_{j_{1}} \cap C_{j_{2}}=\emptyset, \quad j_{1} \neq j_{2} .
$$

Let $h$ be the least common multiple of $\left|C_{1}\right|, \ldots,\left|C_{r}\right|$,

$$
h=\left[\left|C_{1}\right|, \ldots,\left|C_{r}\right|\right] \text {. }
$$

Note that $h$ is of order 2 modulo $m$ (this follows easily, e.g., from Exercise 3, p. 104 in [12]).

Definition 1. The exact exponent in the remainder term in (2) is $\alpha=$ $\alpha(m)$ if

$$
T_{m, a}^{j}(x)=\frac{x}{2 m}+O\left(x^{\alpha+\varepsilon}\right), \quad T_{m, a}^{j}(x)=\frac{x}{2 m}+\Omega\left(x^{\alpha-\varepsilon}\right), \quad \forall \varepsilon>0 .
$$

Our main result is the following.

TheOREM 1 . If $m \geq 3$ is odd, then the exact exponent in the remainder term in (2) is

$$
\alpha=\max _{1 \leq l \leq m-1}\left(1+\frac{1}{h \ln 2} \sum_{k=0}^{h-1}\left(\ln \left|\sin \frac{\pi l 2^{k}}{m}\right|\right)\right) .
$$

Note that, if 2 is a primitive root of an odd prime $p$, then $r=1, h=p-1$. As a corollary of Theorem 1 we obtain the following result.

THEOREM 2. If $p$ is an odd prime for which 2 is a primitive root, then the exact exponent in the remainder term in (2) is

$$
\alpha=\frac{\ln p}{(p-1) \ln 2} .
$$

Theorem 2 generalizes the well-known result for $p=3$ ([11], [2], [1]). Furthermore, we say that 2 is a semiprimitive root modulo $p$ if 2 is of order $(p-1) / 2$ modulo $p$ and the congruence $2^{x} \equiv-1 \bmod p$ is not solvable. E.g., 2 is of order 8 modulo 17 , but the congruence $2^{x} \equiv-1 \bmod 17$ has the solution $x=4$. Therefore, 2 is not a semiprimitive root of 17 . The first 
primes for which 2 is a semiprimitive root are (see [14, A 139035])

$$
7,23,47,71,79,103,167,191,199,239,263, \ldots \text {. }
$$

For these primes we have $r=2$ and $h=(p-1) / 2$. As a second corollary of Theorem 1 we obtain the following result.

THEOREM 3. If $p$ is an odd prime for which 2 is a semiprimitive root, then the exact exponent $\alpha$ in the remainder term in (2) is also given by (9).

We also prove the following lower estimate for $\alpha(m)$.

TheOREM 4. For $m$ odd,

$$
\alpha(m) \geq \frac{\ln m}{r h \ln 2} .
$$

In particular, if $m=p$ is prime, then $r h=p-1$ and

$$
\alpha(p) \geq \frac{\ln p}{(p-1) \ln 2} .
$$

Note that, if Artin's conjecture of the infinity of primes for which 2 is a primitive root is true, then by Theorem 2 ,

$$
\liminf _{p \rightarrow \infty} \alpha(p)=0 .
$$

In Section 2 we provide an explicit formula for $S_{m, a}(x)$, while in Sections 3-4 we prove Theorems $1-4$.

2. Explicit formula for $S_{m, a}(x)$. Let $\lfloor x\rfloor=N$. We have

$$
\begin{aligned}
S_{m, a}(N) & =\sum_{n=0, m \mid n-a}^{N-1}(-1)^{s(n)}=\frac{1}{m} \sum_{t=0}^{m-1} \sum_{n=0}^{N-1}(-1)^{s(n)} e^{2 \pi i(n-a) t / m} \\
& =\frac{1}{m} \sum_{t=0}^{m-1} \sum_{n=0}^{N-1} e^{2 \pi i\left(\frac{t}{m}(n-a)+\frac{1}{2} s(n)\right)} .
\end{aligned}
$$

Note that the interior sum is of the form

$$
\Phi_{a, \beta}(N)=\sum_{n=0}^{N-1} e^{2 \pi i\left(\beta(n-a)+\frac{1}{2} s(n)\right)}, \quad 0 \leq \beta<1 .
$$

Putting

$$
F_{\beta}(N)=e^{2 \pi i \beta a} \Phi_{a, \beta}(N),
$$

we note that $F_{\beta}(N)$ does not depend on $a$.

Lemma 1. If $N=2^{\nu_{0}}+2^{\nu_{1}}+\cdots+2^{\nu_{\sigma}}$ with $\nu_{0}>\nu_{1}>\cdots>\nu_{\sigma} \geq 0$, then

$$
F_{\beta}(N)=\sum_{g=0}^{\sigma} e^{2 \pi i\left(\beta \sum_{j=0}^{g-1} 2^{\nu_{j}}+g / 2\right)} \prod_{k=0}^{\nu_{g}-1}\left(1+e^{2 \pi i\left(\beta 2^{k}+1 / 2\right)}\right) .
$$


Proof. Let $\sigma=0$. Then by (12) and (13),

$$
\begin{aligned}
F_{\beta}(N) & =\sum_{n=0}^{N-1}(-1)^{s(n)} e^{2 \pi i \beta n} \\
& =1-\sum_{j=0}^{\nu_{0}-1} e^{2 \pi i \beta 2^{j}}+\sum_{0 \leq j_{1}<j_{2} \leq \nu_{0}-1} e^{2 \pi i \beta\left(2^{j_{1}}+2^{j_{2}}\right)}-\cdots \\
& =\prod_{k=0}^{\nu_{0}-1}\left(1-e^{2 \pi i \beta 2^{k}}\right),
\end{aligned}
$$

which corresponds to (14) for $\sigma=0$.

Assuming that (14) is valid for every $N$ with $s(N)=\sigma+1$, let us consider $N_{1}=2^{\nu_{\sigma}} b+2^{\nu_{\sigma+1}}$ where $b$ is odd, $s(b)=\sigma+1$ and $\nu_{\sigma+1}<\nu_{\sigma}$. Let

$$
\begin{aligned}
N & =2^{\nu_{\sigma}} b=2^{\nu_{0}}+\cdots+2^{\nu_{\sigma}}, \\
N_{1} & =2^{\nu_{0}}+\cdots+2^{\nu_{\sigma}}+2^{\nu_{\sigma+1}} .
\end{aligned}
$$

Notice that for $n \in\left[0,2^{\nu_{\sigma+1}}\right)$ we have

$$
s(N+n)=s(N)+s(n) .
$$

Therefore,

$$
\begin{aligned}
F_{\beta}\left(N_{1}\right) & =F_{\beta}(N)+\sum_{n=N}^{N_{1}-1} e^{2 \pi i\left(\beta n+\frac{1}{2} s(n)\right)} \\
& =F_{\beta}(N)+\sum_{n=0}^{2^{\nu} \sigma+1-1} e^{2 \pi i\left(\beta n+\beta N+\frac{1}{2}(s(N)+s(n))\right)} \\
& =F_{\beta}(N)+e^{2 \pi i\left(\beta N+\frac{1}{2} s(N)\right)} \sum_{n=0}^{2^{\nu \sigma+1}-1} e^{2 \pi i\left(\beta n+\frac{1}{2} s(n)\right)} .
\end{aligned}
$$

Thus, by (14) and (15),

$$
\begin{aligned}
F_{\beta}\left(N_{1}\right)= & \sum_{g=0}^{\sigma} e^{2 \pi i\left(\beta \sum_{j=0}^{g-1} 2^{\nu_{j}}+g / 2\right)} \prod_{k=0}^{\nu_{g}-1}\left(1+e^{2 \pi i\left(\beta 2^{k}+1 / 2\right)}\right) \\
& +e^{2 \pi i\left(\beta \sum_{j=0}^{\sigma} 2^{\nu_{j}}+(\sigma+1) / 2\right)} \prod_{k=0}^{\nu_{g+1}-1}\left(1+e^{2 \pi i\left(\beta 2^{k}+1 / 2\right)}\right) \\
= & \sum_{g=0}^{\sigma+1} e^{2 \pi i\left(\beta \sum_{j=0}^{g-1} 2^{\nu_{j}}+g / 2\right)} \prod_{k=0}^{\nu_{g}-1}\left(1+e^{2 \pi i\left(\beta 2^{k}+1 / 2\right)}\right) .
\end{aligned}
$$


Formulas (11)-(14) give an explicit expression for $S_{m}(N)$ as a linear combination of products of the form

$$
\prod_{k=0}^{\nu_{g}-1}\left(1+e^{2 \pi i\left(\beta 2^{k}+1 / 2\right)}\right), \quad \beta=t / m, 0 \leq t \leq m-1 .
$$

REMARK 1. One may derive (14) from a very complicated general formula of Gelfond [7]. However, we preferred to give an independent proof.

In particular, if $N=2^{\nu}$, then from (11)-(13) and (15) for

$$
\beta=t / m, \quad t=0,1, \ldots, m-1,
$$

we obtain the known formula (cf. [4]):

$$
S_{m, a}\left(2^{\nu}\right)=\frac{1}{m} \sum_{t=1}^{m-1} e^{-2 \pi i \frac{t}{m} a} \prod_{k=0}^{\nu-1}\left(1-e^{2 \pi i \frac{t}{m} 2^{k}}\right) .
$$

3. Proof of Theorem 1. Consider the equation of order $r$

$$
z^{r}+c_{1} z^{r-1}+\cdots+c_{r}=0
$$

with the roots

$$
z_{j}=\prod_{t \in C_{j}}\left(1-e^{2 \pi i t / m}\right), \quad j=1, \ldots, r .
$$

Notice that for $t \in C_{j}$ we have

$$
\prod_{k=n+1}^{n+h}\left(1-e^{2 \pi i 2^{k} / m}\right)=\left(\prod_{t \in C_{j}}\left(1-e^{2 \pi i t / m}\right)\right)^{h / h_{j}}=z_{j}^{h / h_{j}},
$$

where $h$ is defined by (7). Therefore, for every $t \in\{1, \ldots, m-1\}$, according to (19) we have

$$
\begin{aligned}
& \prod_{k=n+1}^{n+r h}\left(1-e^{2 \pi i t 2^{k} / m}\right) \\
+ & c_{1} \prod_{k=n+1}^{n+(r-1) h}\left(1-e^{2 \pi i t 2^{k} / m}\right)+\cdots+c_{r-1} \prod_{k=n+1}^{n+h}\left(1-e^{2 \pi i t 2^{k} / m}\right)+c_{r}=0 .
\end{aligned}
$$

After multiplication by $e^{-2 \pi i \frac{t}{m} a} \prod_{k=0}^{n}\left(1-e^{2 \pi i t 2^{k} / m}\right)$ and summing over $t=1, \ldots, m-1$, by (18) we find

$$
\begin{aligned}
S_{m, a}\left(2^{n+r h+1}\right)+ & c_{1} S_{m, a}\left(2^{n+(r-1) h+1}\right) \\
& +\cdots+c_{r-1} S_{m, a}\left(2^{n+h+1}\right)+c_{r} S_{m, a}\left(2^{n+1}\right)=0 .
\end{aligned}
$$


Moreover, using the general formulas (11)-(14) for a positive integer $u$, we obtain the equality

$$
\begin{aligned}
S_{m, a}\left(2^{r h+1} u\right)+c_{1} S_{m, a} & \left(2^{(r-1) h+1} u\right) \\
+ & \cdots+c_{r-1} S_{m, a}\left(2^{h+1} u\right)+c_{r} S_{m, a}(2 u)=0 .
\end{aligned}
$$

Putting here

$$
S_{m, a}\left(2^{u}\right)=f_{m, a}(u)
$$

we have

$$
\begin{aligned}
f_{m, a}(y+r h+1) & +c_{1} f_{m, a}(y+(r-1) h+1) \\
& +\cdots+c_{r-1} f_{m, a}(y+h+1)+c_{r} f_{m, a}(y+1)=0,
\end{aligned}
$$

where

$$
y=\log _{2} u \text {. }
$$

The characteristic equation of $(26)$ is

$$
v^{r h}+c_{1} v^{(r-1) h}+\cdots+c_{r-1} v^{h}+c_{r}=0 .
$$

A comparison of (28) and (20)-(21) shows that the roots of (28) are

$$
v_{j, w}=e^{2 \pi i w / h} \prod_{t \in C_{j}}\left(1-e^{2 \pi i t / m}\right)^{1 / h}, \quad w=0, \ldots, h-1, j=1, \ldots, r .
$$

Thus,

$$
v=\max \left|v_{j, l}\right|=2 \max _{1 \leq l \leq m-1}\left(\prod_{k=0}^{h-1}\left|\sin \frac{\pi l 2^{k}}{m}\right|\right)^{1 / h} .
$$

Generally speaking, some numbers in (20) could be equal. In view of (29), the $v_{j, w}$ 's have the same multiplicities. If $\eta$ is the maximal multiplicity, then according to (25) and (27),

$$
S_{m, a}(u)=f_{m, a}\left(\log _{2} u\right)=O\left(\left(\log _{2} u\right)^{\eta-1} u^{\ln v / \ln 2}\right) .
$$

Nevertheless, at least

$$
S_{m, a}(u)=\Omega\left(u^{\ln v / \ln 2}\right) .
$$

Indeed, let, say, $v=\left|v_{1, w}\right|$ and suppose that in the solution of (26) with some natural initial conditions, all coefficients of $y^{j_{1}} v_{1, w}^{y}, j_{1} \leq \eta-1, w=0, \ldots$, $h-1$, are 0 . Then $f_{m, a}(y)$ satisfies a difference equation with the characteristic equation not having roots $v_{1, w}$, and the corresponding relation for $S_{m, a}\left(2^{n}\right)$ (see (23)) has the characteristic equation (19) without the root $z_{1}$. This is impossible since by (18) and (21) we have

$$
S_{m, a}\left(2^{h+1}\right)=\frac{1}{m} \sum_{j=1}^{r} \sum_{t \in C_{j}} e^{-2 \pi i \frac{t}{m} a} \prod_{k=1}^{h}\left(1-e^{2 \pi i \frac{t}{m} 2^{k}}\right)=\frac{1}{m} \sum_{j=1}^{r} \sum_{t \in C_{j}} e^{-2 \pi i \frac{t}{m} a} z_{j}^{h / h_{j}} .
$$


Therefore, the coefficients considered do not all vanish, and (32) follows. Now from (30)-(32) we obtain (8).

REMARK 2. In (8) it is sufficient to let $l$ run over a system of distinct representatives of the cyclotomic cosets $C_{1}, \ldots, C_{r}$ of 2 modulo $m$.

REMARK 3. It is easy to see that there exists $l \geq 1$ such that $\left|C_{l}\right|=2$ if and only if $m$ is a multiple of 3. Moreover, for $l$ we can take $m / 3$. Now from (8), choosing $l=m / 3$, we obtain $\alpha=\lambda=\ln 3 / \ln 4$. This result was obtained in [13] together with estimates of the constants in $S_{m, 0}(x)=O\left(x^{\lambda}\right)$ and $S_{m, 0}(x)=\Omega\left(x^{\lambda}\right)$ which are based on the formula, proved in [13],

$$
S_{m, 0}(x)=\frac{3}{m} S_{3,0}(x)+O\left(x^{\lambda_{1}}\right)
$$

for $\lambda_{1}=\lambda_{1}(m)<\lambda$ and Coquet's theorem [2].

EXAmPle 1 . Let $m=17, a=0$. Then $r=2, h=8$,

$$
C_{1}=\{1,2,4,8,16,15,13,9\}, \quad C_{2}=\{3,6,12,7,14,11,5,10\} .
$$

The calculation of

$$
\alpha_{l}=1+\frac{1}{8 \ln 2} \sum_{k=0}^{17}\left(\ln \left|\sin \frac{\pi l 2^{k}}{17}\right|\right)
$$

for $l=1$ and $l=3$ gives

$$
\alpha_{1}=-0.12228749 \ldots, \quad \alpha_{3}=0.63322035 \ldots
$$

Therefore by Theorem $1, \alpha=0.63322035 \ldots$ Moreover, we will prove that

$$
\alpha=\frac{\ln (17+4 \sqrt{17})}{\ln 256} \text {. }
$$

Indeed, according to (23), for $n=0$ and $n=1$ we obtain the system $\left(S_{17,0}=S_{17}\right)$ :

$$
\left\{\begin{array}{l}
c_{1} S_{17}\left(2^{9}\right)+c_{2} S_{17}(2)=-S_{17}\left(2^{17}\right), \\
c_{1} S_{17}\left(2^{10}\right)+c_{2} S_{17}\left(2^{2}\right)=-S_{17}\left(2^{18}\right) .
\end{array}\right.
$$

By direct calculations we find

$$
\begin{aligned}
& S_{17}(2)=1, \quad S_{17}\left(2^{2}\right)=1, \quad S_{17}\left(2^{9}\right)=21, \\
& S_{17}\left(2^{10}\right)=29, \quad S_{17}\left(2^{17}\right)=697, \quad S_{17}\left(2^{18}\right)=969 .
\end{aligned}
$$

Solving (33) we obtain

$$
c_{1}=-34, \quad c_{2}=17
$$

Thus, by (23) and (24),

$$
\begin{aligned}
S_{17}\left(2^{n+17}\right) & =34 S_{17}\left(2^{n+9}\right)-17 S_{17}\left(2^{n+1}\right), \quad n \geq 0, \\
S_{17}\left(2^{17} x\right) & =34 S_{17}\left(2^{9} x\right)-17 S_{17}(2 x), \quad x \in \mathbb{N} .
\end{aligned}
$$


Putting furthermore

$$
S_{17}\left(2^{x}\right)=f(x)
$$

we have

$$
f(y+17)=34 f(y+9)-17(y+1),
$$

where $y=\log _{2} x$. Hence,

$$
f(x)=O\left((17+4 \sqrt{17})^{x / 8}\right),
$$

that is

$$
S_{17}(x)=O\left((17+4 \sqrt{17})^{\frac{1}{8} \log _{2} x}\right)=O\left(x^{\alpha}\right),
$$

where

$$
\alpha=\frac{\ln (17+4 \sqrt{17})}{\ln 256}=0.633220353 \ldots .
$$

\section{Proofs of Theorems 2-4}

Proof of Theorem 2. By the assumptions of Theorem 2 we have $r=1$ and $h=p-1$. Using (8) we have

$$
\begin{aligned}
\alpha & =1+\frac{1}{(p-1) \ln 2} \ln \prod_{k=0}^{p-2}\left|\sin \frac{\pi 2^{k}}{p}\right| \\
& =1+\frac{1}{(p-1) \ln 2} \ln \prod_{l=1}^{p-1} \sin \frac{\pi l}{p} .
\end{aligned}
$$

Furthermore, using the identity

$$
\prod_{l=1}^{p-1} \sin \frac{l \pi}{p}=\frac{p}{2^{p-1}}
$$

([6, p. 378] for example), we find

$$
\alpha=1+\frac{1}{(p-1) \ln 2}(\ln p-(p-1) \ln 2)=\frac{\ln p}{(p-1) \ln 2} .
$$

REMARK 4. In this case, (24) has the simple form

$$
S_{p, a}\left(2^{p} u\right)+c_{1} S_{p, a}(2 u)=0 .
$$

Since in the case of $a=0$ or 1 we have

$$
S_{p, a}(2)=(-1)^{s(a)},
$$

while in the case of $a \geq 2$,

$$
S_{p, a}(2 a)=(-1)^{s(a)},
$$

putting

$$
u= \begin{cases}1, & a=0,1 \\ a, & a \geq 2\end{cases}
$$


we find

$$
c_{1}=(-1)^{s(a)+1} \begin{cases}S_{p, a}\left(2^{p}\right), & a=0,1 \\ S_{p, a}\left(a 2^{p}\right), & a \geq 2 .\end{cases}
$$

In particular, if $p=3$ and $a=2$ we have $c_{1}=S_{3,2}(16)=-3$ and

$$
S_{3,2}(8 u)=3 S_{3,2}(2 u) \text {. }
$$

Proof of Theorem 3. By the assumptions of Theorem 3 we have $r=2$ and $h=(p-1) / 2$, so that cyclotomic cosets of 2 modulo $p$ satisfy

$$
C_{1}=-C_{2} \text {. }
$$

Therefore, in (8) we obtain the same values for $l_{1}=1$ and $l_{2}=p-1$. Thus,

$$
\alpha=1+\frac{2}{(p-1) \ln 2} \ln \left(\prod_{l=1}^{p-1} \sin \frac{\pi l}{p}\right)^{1 / 2}=\frac{\ln p}{(p-1) \ln 2}
$$

Proof of Theorem 4. According to (19)-(20),

$$
c_{r}=(-1)^{r} \prod_{j=1}^{r} \prod_{t \in C_{j}}\left(1-e^{2 \pi i t / m}\right)=(-1)^{r} \prod_{t=1}^{m-1}\left(1-e^{2 \pi i t / m}\right) .
$$

Thus, using (38) we have

$$
\left|c_{r}\right|=2^{m} \prod_{t=1}^{m-1} \sin \frac{\pi t}{m}=m .
$$

Consequently, by (29),

$$
\prod_{j=1}^{r}\left|v_{j, w}\right|=m^{1 / h}, \quad w=0,1, \ldots, h-1 .
$$

Therefore,

$$
v=\max \left|v_{j, w}\right| \geq m^{1 / r h}
$$

and Theorem 4 follows.

Using Theorems 1-3, in particular, we find

$$
\begin{aligned}
\alpha(3) & =0.7924 \ldots, & \alpha(5) & =0.5804 \ldots, & \alpha(7) & =0.4678 \ldots, \\
\alpha(11) & =0.3459 \ldots, & \alpha(13) & =0.3083 \ldots, & \alpha(17) & =0.6332 \ldots, \\
\alpha(19) & =0.2359 \ldots, & \alpha(23) & =0.2056 \ldots, & \alpha(29) & =0.1734 \ldots, \\
\alpha(31) & =0.6358 \ldots, & \alpha(37) & =0.1447 \ldots, & \alpha(41) & =0.4339 \ldots, \\
\alpha(43) & =0.6337 \ldots, & \alpha(47) & =0.1207 \ldots, & &
\end{aligned}
$$

Acknowledgments. The author is grateful to Professor Daniel Berend (Department of Mathematics and of Computer Science, Ben-Gurion University) for his significant influence on the final version of this paper. 
The article is partly supported by a grant of the Kamea Foundation of Israeli Ministry of Absorption.

\section{References}

[1] J.-P. Allouche and J. Shallit, Automatic Sequences: Theory, Applications, Generalizations, Cambridge Univ. Press, 2003.

[2] J. Coquet, A summation formula related to the binary digits, Invent. Math. 73 (1983), 107-115.

[3] C. Dartige et G. Tenenbaum, Sommes des chiffres de multiples d'entiers, Ann. Inst. Fourier (Grenoble) 55 (2005), 2423-2474.

[4] M. Drmota and M. Skałba, Rarified sums of the Thue-Morse sequence, Trans. Amer. Math. Soc. 352 (1999), 609-642.

[5] M. Drmota and T. Stoll, Newman's phenomenon for generalized Thue-Morse sequences, Discrete Math. 308 (2008), 1191-1208.

[6] G. Freiman and H. Halberstam, On a product of sines, Acta Arith. 49 (1988), 377385.

[7] A. O. Gelfond, Sur les nombres qui ont des propriétés additives et multiplicatives données, ibid. 13 (1968), 259-265.

[8] F. J. MacWilliams and N. J. A. Sloane, The Theory of Error-Correcting Codes, Elsevier/North-Holland, 1977.

[9] C. Mauduit et J. Rivat, Sur un problème de Gelfond: la somme des chiffres des nombres premiers, Ann. of Math., to appear.

[10] C. Mauduit and A. Sárközy, On the arithmetic structure of sets characterized by sum of digits properties, J. Number Theory 61 (1996), 25-38.

[11] D. J. Newman, On the number of binary digits in a multiple of three, Proc. Amer. Math. Soc. 21 (1969), 719-721.

[12] D. Redmond, Number Theory: an Introduction, Dekker, New York, 1996.

[13] V. Shevelev, Generalized Newman phenomena and digit conjectures on primes, Int. J. Math. Math. Sci. 2008, ID 908045.

[14] N. J. A. Sloane, The On-Line Encyclopedia of Integer Sequences, http://www. research.att.com.

[15] T. Stoll, Multi-parametric extensions of Newman's phenomenon, Integers 5 (3) (2005), \#14.

Department of Mathematics

Ben-Gurion University of the Negev

Beer-Sheva 84105, Israel

E-mail: shevelev@bgu.ac.il

Received on 10.6.2008

and in revised form on 1.8.2008 